\title{
IMPLEMENTATION OF MPC ON A DEETHANIZER AT KÅRSTØ GAS PLANT
}

\author{
Elvira Marie B. Aske ${ }^{*, * *}$, Stig Strand ${ }^{* *}$ \\ Sigurd Skogestad ${ }^{*, 1}$ \\ * Department of Chemical Engineering, Norwegian \\ University of Science and Technology, N-7491 Trondheim, \\ Norway \\ ${ }^{* *}$ Statoil REDD, Process Control, N-7005 Trondheim, \\ Norway
}

\begin{abstract}
Model predictive control (MPC) is implemented on several distillation columns at the Kårst $\varnothing$ gas processing plant, Norway. The paper describes the procedure in the implementation of MPC at a deethanizer using the SEPTIC* MPC tool, including design, estimator development, model development and tuning. For the deethanizer, the variance in the product quality has been reduced with about $50 \%$. The number of flaring episodes has also been reduced. An increase in impurities has not been challenged yet, so the average reflux flow and steam consumption to feed ratios are almost unaltered. Copyright (C)2005 IFAC
\end{abstract}

* SEPTIC: Statoil Estimation and Prediction Tool for Identification and Control

Keywords: Model based control, distillation columns, implementation, dynamic modelling, estimators.

\section{INTRODUCTION}

\subsection{Plant description}

The Kårstø gas processing plant plays a key role in the transport and treatment of gas and condensate from central parts of the Norwegian continental shelf. This plant receives rich gas and unstabilized condensate through pipelines and separates the feed into its various components. The products from the plant are sales gas, which is exported in pipelines, and ethane, propane, iso-, normal butane, naphtha and condensate, which are exported by ships. The rich gas processing design capacity at Kårst $\varnothing$ is today at $74 \mathrm{MSm} 3 / \mathrm{d}$. The facility had 575 ship calls in 2002 to load the liquid products,

1 Corresponding author: skoge@chemeng.ntnu.no and is one of the largest producers of liquefied petroleum gases (LPG) in the world.

\subsection{Model predictive control}

MPC is sometimes defined as the family of controllers where there is a direct use of an explicit and separately identifiable model, where the model provides predictions of the process response to future changes in the manipulative variables and to predicted process disturbances (Garcia et al., 1989). In practice, MPC is characterized by its ability to handle constraints in both manipulated and controlled variables. MPC techniques provides the only methodology to handle constraints in a systematic way during the design and implementation of the controller. Moreover, in its most general form MPC is not restricted in terms of 
the model, objective function and/or constraint functionality. These are the primary reasons for the success of these techniques in numerous applications in the chemical process industries (Garcia et al., 1989; Qin and Badgwell, 2003).

The most important issues for the Kårst $\varnothing$ processing plant are regularity and capacity, to avoid being a bottleneck in the large gas transportation system in the Norwegian Sea. While several extension projects gradually increase the plant size and complexity, the resulting regularity challenges are met with MPC implementation. Moreover, large value creations take place, and pushing the capacity limits requires a control tool like MPC to handle the varying set of active constraints.

\section{SEPTIC MPC}

SEPTIC is an in-house software system for MPC, real-time optimization (RTO), dynamic process simulation for simpler case studies, and off- and on-line parameter estimation in first principle based process models. At Kårst $\varnothing$, SEPTIC was selected as a tool for MPC. The MPC issues of SEPTIC are described by Strand and Sagli (2003).

Currently, most SEPTIC MPC applications in Statoil use experimental SISO step response models. SEPTIC is also capable of running generally non-linear models implemented in a compact model object. However, the SISO models represent to a large extent the process dynamics sufficiently accurate to achieve good controller performance.

\section{The SEPTIC MPC is configured with}

- controlled variables (CV), specified with setpoint (SP), high limit and low limit,

- manipulated variables (MV), specified with rate of change, high and low limit and ideal value (IV),

- disturbance variables (DV).

The control specifications are explicitly prioritized by:

(1) MV rate of change limits

(2) MV high and low limits

(3) CV hard constraints, hardly ever used

(4) CV setpoints, CV high and low limits and MV ideal values with priority level 1

(5) CV setpoints, CV high and low limits and MV ideal values with priority level $n$

(6) CV setpoints, CV high and low limits and MV ideal values with priority level 99

MV rate of change and MV high and low limits are always activated and respected unless there is a dynamic conflict between those two specifications. Then a sequence of steady-state quadratic programs is solved to respect the remaining specifications 3) - 6), giving the achievable steady-state targets. The control specifications are adjusted accordingly for the dynamic optimization problem.

\section{DEETHANIZER MPC}

The implementation of MPC for the Sleipner train deethanizer is described in the following chapter.

\subsection{Column description}

The deethanizer has 34 trays, a partial condenser with propane coolant, a reflux drum, and a reboiler with LP steam as heating medium. The gas from the reflux drum goes to the steam boilers as fuel gas, and the liquid splits to reflux and distillate. The column feed is the top product from two stabilizers that consists of butane and lighter components. The feed passes through the gas dryers to remove water before it enters the column.

The deethanizer basic control structure can be summarized as follows:

- Reflux drum level control with distillate

- Reflux flow control

- Column bottom level control

- Tray 1 temperature control with condensate

- LP steam pressure control

- Column pressure control by reflux drum gas valve

The column including the basic control structure is displayed in figure 1 . The performance to the PID controllers around the column is verified and tuned if necessary before any MPC modelling take place.

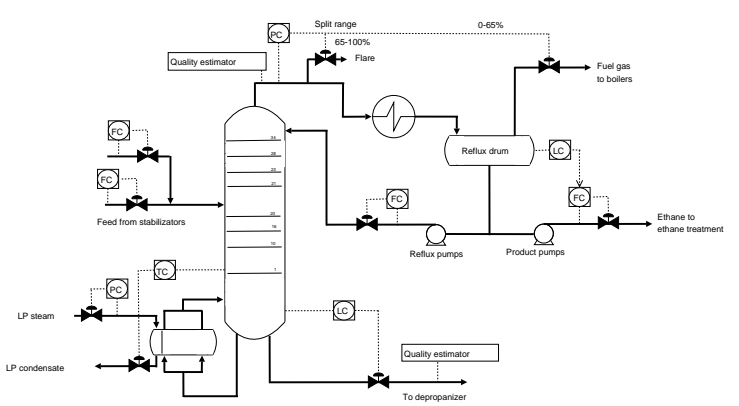

Fig. 1. The deethanizer including the basic control

There are three main disturbances to consider in operation. First, the feed rate may be reduced to the half of its nominal value in less than 15 minutes. This occurs when one of the two stabilizers are taken out of production. Second, the feed flow composition may change. There are analyzers on both feed streams, but the sampling time is about 15 minutes, so the column responds 
to the variations before the analyzers. The third disturbance is feed temperature variations due to the 1-2 days gas drier regeneration cycle.

\section{2 $M P C$ design}

The MPC design starts with MV, CV and DV selection. The system components are the column, condenser, reflux drum and reboiler, while the input and output streams are feed and products. The main control objective is to control the quality of the top and bottom streams, by manipulating boil-up and reflux flow.

The temperature controller set point is selected as an MV. An option is to manipulate the steam flow, which is a direct manipulation on the energy input. However, the original configuration is kept and leaves the basic control scheme unchanged for the operators. Manipulating the temperature controller set point requires that the temperature controller dynamics must be included in the MPC models.

Also, the column must be kept under surveillance to avoid overloading. The differential pressure is a good indicator for flooding (Kister, 1990), but is not measured for the actual column. In addition, limitations in the basic level control and in the process equipment must be considered. The pressure controller output is included as a $\mathrm{CV}$ to avoid the flare valve opening when the controller exceeds $65 \%$.

Only the feed flow is included as a DV in the MPC. The unmeasured feed composition changes are suppressed by the MPC feedback action. The feed temperature is measured and may be used as a DV if some special gas drier considerations are made.

Manipulating the column pressure is a trade off between energy savings and flooding limit. The pressure is not included as an MV, but could have lead to a more optimal operation of the column.

The steady state gain between the reflux flow and the bottom quality is positive. The temperature controller is in closed loop and to some extent compensates for the reflux flow. However, if the temperature controller was located higher in the column, the steady state gain may have been negative. The other steady-state gains are as expected. The deethanizer MPC design including the steady state gains is summarized in table 1.

The top and bottom product qualities must be measured in some way. The top quality is expressed in propane mol\% in ethane (C3 IN C2), whereas the bottom quality is expressed in ethane mol\% in propane (C2 IN C3). There are on-line gas chromatographs (GC) at the deethanizer dis- tillate and at the depropanizer distillate. The GC sample rate is 10 minutes, which from a control point of view is too infrequent. In addition, the GC is occasionally inoperative due to maintenance. The product qualities are therefore estimated by the temperature profile in the column. A more detailed description of the quality estimators is found in section 3.3.

The CV prioritizing for the deethanizer application is as follows:

(1) High and low limit pressure controller output, high limit top and bottom quality

(2) Set point top and bottom quality

where 1 is the highest priority. The priority list leads to relaxation of the quality set points when the application predicts on one of the limits to the pressure controller output.

Application subgrouping must be considered in the design. In this MPC, the top quality and the reflux flow are in one subgroup and both are critical variables. The bottom quality and the temperature are in another subgroup and both are critical members of the group. The pressure controller output and the column feed are members of both subgroups but are stated as noncritical members. This means that top quality is still allowed to be controlled with reflux but not with temperature if bottom quality is deactivated and vice versa.

\subsection{Obtaining estimators}

The deethanizer data history had sufficient variance in the product qualities, so no test period was needed to enrich the data. The calibration data represented a two month period with 20 minute averages.

The deethanizer and depropanizer GC values are time shifted 10 and 25 minutes respectively, to account for sampling delay and process dynamics.

Distillation columns are known to be strongly nonlinear due to the vapor-liquid equilibrium (VLE). Logarithmic compositions reduce the nonlinearity and the behavior becomes much less dependent on the operation point (Skogestad, 1997). Different quality transformations were tried for the estimator calibration, and the square root gave the best fit.

The least squares regression gave that to describe the top product quality only the tray 28 and top temperatures are needed, whereas the tray 10 and bottom temperatures are needed for the bottom product quality. 
Table 1. The selected variables in the MPC including steady state gain

\begin{tabular}{cccc} 
& MV:Reflux & MV: Temperature & DV:Column Feed \\
\hline CV: C3 in C2 & - & + & + \\
CV: C2 in C3 & + & - & + \\
CV: PC output & + & - & 0 \\
\hline
\end{tabular}

\subsection{Dynamic modelling}

The deethanizer modelling took two days with step testing, with the MV steps and DV (feed rate) variations shown in figure 2 .

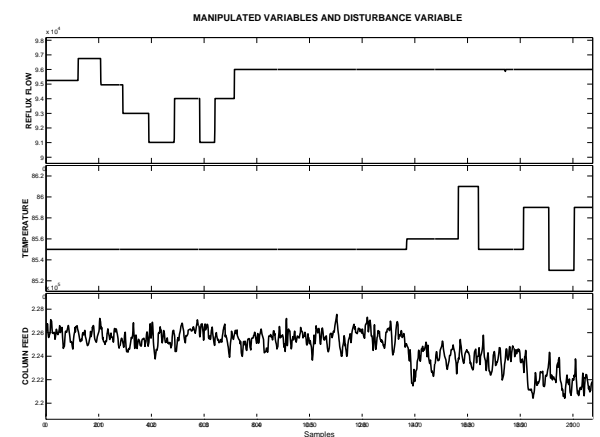

Fig. 2. Step test period for MVs and DV

The resulting CV's are displayed in figure 3.

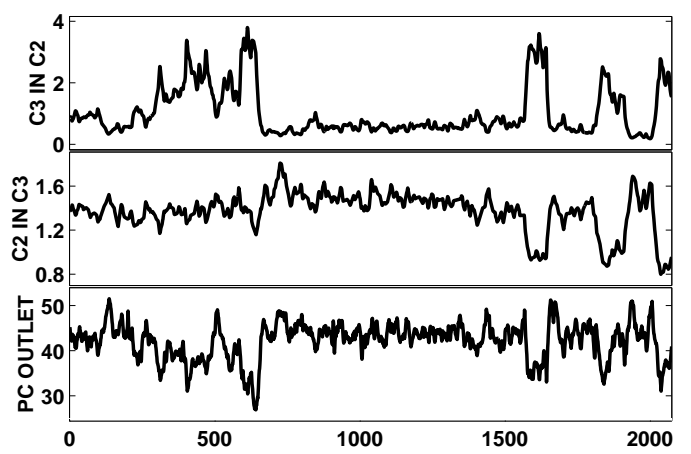

Fig. 3. Resulting CV's from the step test period

The GC is compared with the estimator and shows a satisfactory match, illustrated by the top quality in figure 4.

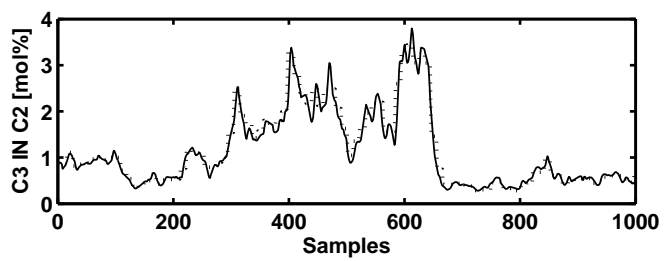

Fig. 4. Top quality, GC (dotted) versus estimator (solid)

The dynamic models are identified by Tai-Ji ID (Zhu, 1998). The Tai-Ji ID identification is based on the asymptotic method (ASYM), which calculates time domain parametric models using frequency domain criterion. The step response models from the Tai-Ji ID tool is displayed in figure 5.
The grading A to D is determined from the upper error bounds in a frequency plot. The steady state gains in the models are as expected, except the column feed influence on the top quality that turns out to be negative. A positive steady state gain effect for this model is found from data with more variations in the feed. The model fit is displayed in figure 6 .

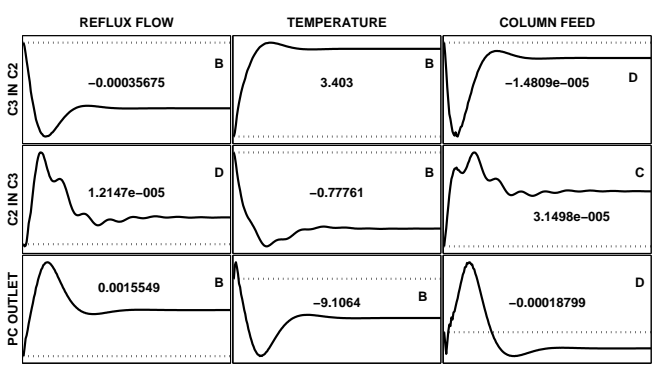

Fig. 5. Step response models for the deethanizer application

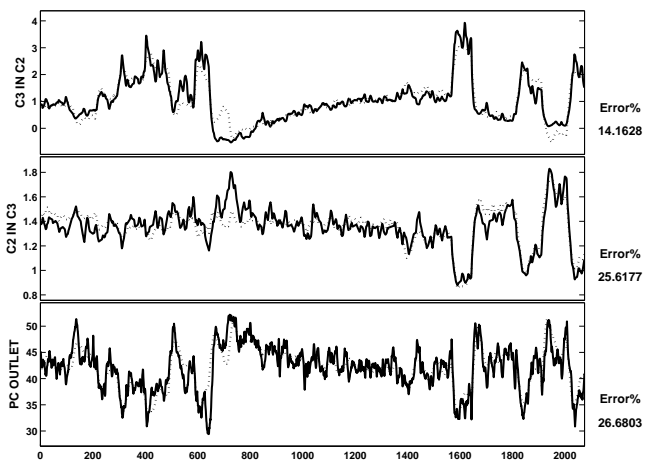

Fig. 6. The model fit. Measured CV's (solid) and simulated CV's (dashed)

Experience from other MPC applications have shown that using the logarithmic qualities gives better adaption to step response models. The logarithmic composition is defined as the logarithm between the ratio of the key components, Skogestad (1997), and is written as

$$
X=\log \frac{0.01 \cdot y}{1-0.01 \cdot y}
$$

where $y$ is the impurity component in $\mathrm{mol} \%$.

The step response models and the model fit of the transformed CV's are displayed in figure 7 and 8 respectively.

The improvement by using logarithmic quality is not that clear in this application. There is 


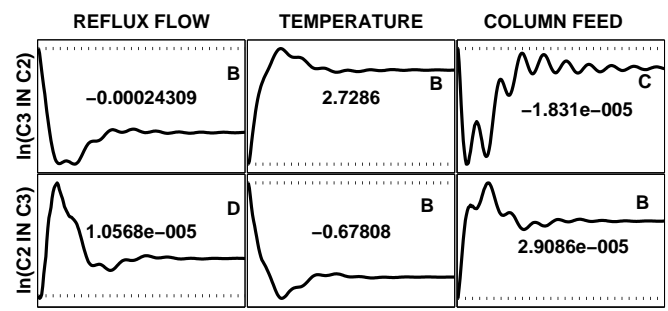

Fig. 7. Step response models with logarithmic transformation of the qualities

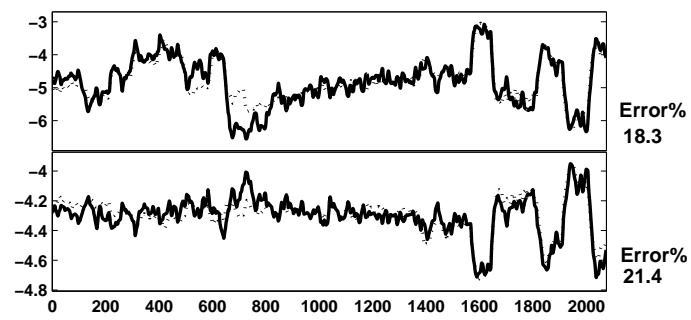

Fig. 8. The logaritmic model fit. Measured CV's (solid) and simulated CV's (dashed)

reduced error in the models between the logarithmic qualities versus the column feed, leads to an improvement from $\mathrm{C}$ to $\mathrm{B}$ model for the top and $\mathrm{D}$ to $\mathrm{C}$ model for the bottom, indicating a better initial response. Changes in reflux have a small effect on the bottom quality, and the identification found only a D model in both cases. The frequency plot of the error bounds show a acceptable initial response, which is caused by the temperature controller do not compensate for the reflux change immediately, so the D model is kept in the application.

The models between the CV's and the column feed are verified through a new data set with more variation in the feed. In the new models from column feed, the steady state gain for the top quality and the pressure controller outlet changed sign. The column feed have a small influence on the pressure controller outlet in general so the model is omitted from the application.

\subsection{MPC tuning}

Several tuning parameters must be decided to obtain a rational use of the MV's to reach the control targets. The available set of SEPTIC MPC tuning parameters are:

- $C V$ and $M V$ span: internal scaling reflecting the "acceptable" standard deviation of each variable

- $C V$ Fulf: setpoint deviation penalty

- $M V$ Fulf: ideal value deviation penalty

- CV HighPnlty/LowPnlty: high and low limit violation penalty

- $C V$ SetpTref: time constant for first order low pass filtering of set point changes
- $C V$ ConsTfilt: time constant for first order low pass filtering of high and low limit changes

- $M V$ MovePnlty: change penalty

- MV MaxUp/MaxDown: rate of change limits

- $M V$ IvROC: desired rate of change for IV fulfillment

All penalties are quadratic, including the ones for deviation, violation and move penalty.

A summary of the MPC tuning parameters are given in table 2. The HighPnlty and LowPnlty for the pressure controller output are lower than for the qualities to avoid too aggressive use of the MV's when pressure controller outlet operates close to its limits. The scaling have already proportionate the variables, so the MovePnlty parameter is set to 1. SetpTref and Cons Tfilt is not used in the application. Also typical operation values is listed in table 2. The qualities are specified with a set point value and a high limit value, while the pressure controller output is specified with a high limit and a low limit. The bottom quality high limit is lower than the product specification because of too high ethane content in propane leads to condensation problems in the depropanizer condenser. The pressure controller output high limit is the limitations in the fuel gas system whereas the low limit is introduced to provide a minimum fuel gas stream.

At last, the parameters that specify the model updating are determined. The bottom quality has some noise and the deviation between the model and the $\mathrm{CV}$ is filtered through a 2 minutes low pass filter. Both the top quality and the pressure controller outputs have non-modeled disturbances that influence on the variables. Letting the MV's react fast suppresses these disturbances, so both variables have a first order prediction of the disturbances with 5 minutes time constant. The cost is a more aggressive use of the reflux flow.

\section{RESULTS FROM IMPLEMENTATION}

\subsection{Column operation without $M P C$}

As apposed to other distillation columns at Kårstø, the deethanizer did not operate with particularly high purity in both ends. However, the deethanizer is one of the most sensitive columns with respect to disturbances and changes in reflux flow and boil-up. The basic control scheme gave large variations in product quality due to feed disturbances.

Finding the right combination of temperature set point and reflux flow rate was not easy. This combination changes with feed flow and feed composition, so the operator must be awake and adjust 
Table 2. Typical operation values and MPC tuning parameters for the deethanizer,

$C V$ priority level in parenthesis

\begin{tabular}{|c|c|c|c|c|c|c|c|c|c|}
\hline Variable & $\begin{array}{l}\mathrm{SP} / \\
\mathrm{IV}\end{array}$ & $\begin{array}{l}\text { High } \\
\text { Limit }\end{array}$ & $\begin{array}{l}\text { Low } \\
\text { Limit }\end{array}$ & Span & Fulf & HighPnlty & LowPnlty & $\begin{array}{l}\text { Move- } \\
\text { Pnlty }\end{array}$ & $\begin{array}{l}\text { MaxUp/ } \\
\text { MaxDown }\end{array}$ \\
\hline CV:C3 in C2 [mol\%] & $1.2(2)$ & $4(1)$ & & 0.3 & 0.5 & 5 & & & \\
\hline $\mathrm{CV}: \mathrm{C} 2$ in $\mathrm{C} 3$ [mol\%] & $1.2(2)$ & $2.5(1)$ & & 0.3 & 0.5 & 5 & & & \\
\hline CV:PC output [\%] & & $60(1)$ & $15(1)$ & 1 & & 2.5 & 2.5 & & \\
\hline MV:Reflux flow $[\mathrm{kg} / \mathrm{h}]$ & & 110000 & 55000 & 2000 & & & & 1 & $2000 /-500$ \\
\hline MV:Temperature $\left[{ }^{\circ} \mathrm{C}\right]$ & & 86.3 & 84.5 & 0.2 & & & & 1 & $0.15 /-0.15$ \\
\hline
\end{tabular}

the temperature and the reflux flow several times during a shift.

\subsection{Column operation with MPC}

A 20 days period with 20 minutes interval have been sampled, to compare operation before and after MPC implementation. The most distinctive improvement is the variance in the product qualities. The standard deviation for the top product is reduced with $46 \%$ for the collected data series, whereas the standard deviation for the bottom product is reduced with $56 \%$. The top and bottom quality without and with MPC operation is displayed in figure 9 .
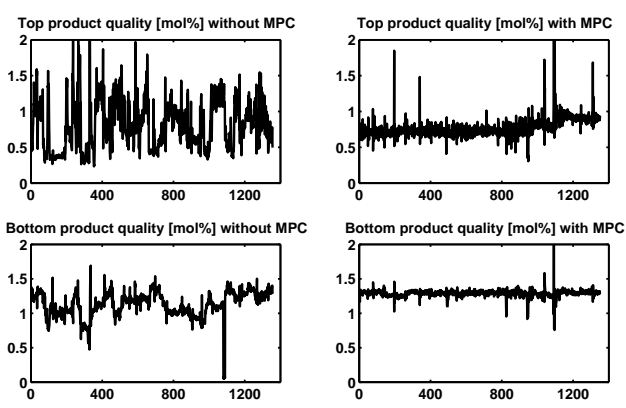

Fig. 9. Product quality from the column without (left) and with(right) MPC

The product qualities have not been changed significantly. The impurities can be increased 1-1.5 mol\%, but the limits have not been challenged yet. Therefore the average changes in reflux flow and steam consumption are small. From the data period, the reflux flow per unit feed is unaltered. The steam consumption per unit feed has decreased with $2 \%$. The average bottom impurity is slightly higher, which can explain the steam consumption reduction.

With too much methane in the feed, flaring is unavoidable since the fuel gas system has limited capacity. However, data from a two months period indicates a 20-40\% flaring frequency reduction and the flaring episodes have most often a shorter duration.

\section{CONCLUSIONS}

A successful MPC implementation at the Kårst $\varnothing$ gas processing plant has been described in detail. Reduced variance in the product qualities and less flaring have been obtained. Also the opportunity to specify the product qualities directly is an advantage gained with MPC. The product qualities have not been changed significantly after implementation of MPC and therefore the average reflux flow and steam consumption to feed ratios are almost unaltered.

\section{ACKNOWLEDGMENT}

The authors will thank the personnel working in the "Optimization project" in Statoil. We would also thank Gassco for financial support.

\section{REFERENCES}

Garcia, C.E., D.M. Prett and M. Morari (1989). Model predictive control: Theory and practice - a survey. Automatica 25(3), 335-348.

Kister, Henry Z. (1990). Distillation Operation. McGraw Hill, NY, USA.

Qin, S.J. and T.A. Badgwell (2003). A survey of industrial model predictive control technology. Control Engineering Practice 11, 733764.

Skogestad, S. (1997). Dynamics and control of distillation columns - a critical survey. Modeling, Identification and Control 18(3), 177-217.

Strand, S. and J.R. Sagli (2003). MPC in Statoil - advantages with in-house technology. $A D$ CHEM 2003, International Symposium on Advanced Control of Chemical Processes, Hong Kong, January 11-14, 2004 pp. 97-103.

Zhu, Yucai (1998). Multivariable process identification for MPC: the asymptotic method and its applications. Journal of Process Control 8(2), 101-115. 